\title{
Towards a theory of baryon resonances
}

\author{
Ulf-G. Meißner ${ }^{1,2,3, *}$ \\ ${ }^{1}$ Helmholtz-Institut für Strahlen- und Kernphysik, Bethe Center for Theoretical Physics and Center for Science and Thought, \\ Universität Bonn, D-53115 Bonn, Germany \\ ${ }^{2}$ Institut für Kernphysik (Theorie), Institute for Advanced Simulation (IAS-4), and Jülich Center for Hadron Physics, \\ Forschungszentrum Jülich, D-52425 Jülich, Germany \\ ${ }^{3}$ Ivane Javakhishvili Tbilisi State University, 0186 Tbilisi, Georgia
}

\begin{abstract}
In this talk, I discuss methods that allow for a systematic and model-independent calculation of the hadron spectrum. These are lattice QCD and/or its corresponding Effective Field Theories. Assorted results are shown and I take the opportunity to discuss some misconceptions often found in the literature.
\end{abstract}

\section{Introduction: QCD and excited states}

QCD is a remarkable theory. Although its Lagrangian can be written in a line

$$
\mathcal{L}_{\mathrm{QCD}}=-\frac{1}{4} G_{\mu \nu}^{a} G^{\mu \nu, a}+\sum_{f} \bar{q}_{f}(i \not D-\mathcal{M}) q_{f}+\ldots,
$$

its fundamental fields, the quarks and gluons, have never been observed in isolation but only appear as constituents of the strongly interacting particles, hadrons and nuclei. In Eq. (1), $D_{\mu}=\partial_{\mu}-i g A_{\mu}^{a} \lambda^{a} / 2$ is the gauge-covariant derivative, $A_{\mu}^{a}(a=1, \ldots, 8)$ the gluon field, $G_{\mu v}^{a}=$ $\partial_{\mu} A_{v}^{a}-\partial_{v} A_{\mu}^{a}-i g\left[A_{\mu}^{b}, A_{v}^{c}\right]$ the gluon field strength tensor, $g$ is the SU(3) gauge coupling, $q_{f}$ a quark spinor of flavor $f(f=u, d, s, c, b, t), \mathcal{M}$ is the diagonal quark mass matrix and the ellipsis stand for gauge-fixing and CP-violating terms not considered here. The quarks come in two types, the light $(u, d, s)$ and heavy $(c, b, t)$ quark flavors, where light and heavy refers to the QCD scale $\Lambda_{\mathrm{QCD}} \simeq 210 \mathrm{MeV}$ (for $N_{f}=5, \overline{M S}, \mu=2 \mathrm{GeV}$ ). In the absence of the quark masses, $\Lambda_{\mathrm{QCD}}$ is the only dimensionful parameter in QCD that is generated by dimensional transmutation through the running of the strong coupling $\alpha_{s}=g^{2} / 4 \pi$.

The Lagrangian of QCD allows us to define two special limits, in which the theory can be analyzed in terms of appropriately formulated effective field theories (EFTs). In the light quark $(f=u, d, s)$ sector, the effective Lagrangian can be written in terms of left- $\left(q_{L}\right)$ and righthanded $\left(q_{R}\right)$ quark fields, such that

$$
\mathcal{L}_{\mathrm{QCD}}=\bar{q}_{L} i \not D q_{L}+\bar{q}_{R} i \not D q_{R}+O\left(m_{f} / \Lambda_{\mathrm{QCD}}\right)
$$

As can be seen, left- and right-handed quarks decouple, which is reflected in the chiral symmetry. It is explicitly broken by the finite but small quark masses $m_{f}$. Furthermore, chiral symmetry is spontaneously broken, leading to

*e-mail: meissner@hiskp.uni-bonn.de the eight pseudo-Goldstone bosons, the pions, the kaons and the eta. These are indeed the lightest hadrons. The pertinent EFT is chiral perturbation theory (CHPT).

Matters are very different for the heavy $c$ and $b$ quarks, where the leading order Lagrangian takes the form

$$
\mathcal{L}_{\mathrm{QCD}}=\bar{Q}_{f} i v \cdot D Q_{f}+O\left(\Lambda_{\mathrm{QCD}} / m_{f}\right),
$$

with $v$ the four-velocity of the heavy quark and $Q_{f}$ denotes a quark spinor of flavor $f(f=c, b)$. Note that to leading order, this Lagrangian is independent of quark spin and flavor, which leads to $S U(2)$ spin and $S U(2)$ flavor symmetries (HQSS and HQFS, respectively). The pertinent EFT to analyze the consequences is heavy quark effective field theory (HQEFT), which comes in different manifestations. Finally, in heavy-light systems, where heavy quarks act as matter fields coupled to the light pions, one can combine CHPT and HQEFT.

There are various reasons to consider excited states. First, the spectrum of QCD is arguably its least understood feature. This is often phrased in terms of questions like: Why do we observe almost only $q q q$ and $\bar{q} q$ states? What is the nature of the XYZ and other "exotic" states? Where are the glueballs predicted by QCD? Note that I put the word exotic in quotation marks, because this usually refers to states that can not be described within the (conventional) quark model. However, it is very obvious, but not accounted for by many, that the quark model is much too simple, particularly in the sector of the light quarks. E.g. it does not account for a whole class of important players in the hadron spectrum, the so-called hadronic molecules. These also provide the bridge to nuclear physics, because hadrons and nuclei are just different manifestations of structure formation in QCD which are intimately linked and should be considered together. It is also important that high-precision data for spectrum studies have been and will be produced with ELSA at Bonn, MAMI at Mainz, CEBAF at Jefferson Lab, the LHCb experiment at CERN, the BESIII experiment at the BEPCII, 
GlueX at Jefferson Lab and in the future with PANDA at FAIR and other labs worldwide. These data clearly pose a challenge for any theoretical approach.

In what follows, I discuss theoretical approaches that will eventually unravel the physics behind the QCD spectrum. To give a wider perspective, I present results for mesons and baryons, although the center of attention of this workshop clearly is the excitation spectrum of the nucleon. Nevertheless, the QCD spectrum should be seen in a broader perspective. To be more precise, I only consider methods that are

\section{- model-independent,}

- can be systematically improved, and

- allow for uncertainty estimates.

If one of these conditions is not fulfilled, a given method will not be considered further. In particular, I eschew models here. So that leaves us with lattice QCD (LQCD) and EFTs or combinations thereof. LQCD can get groundstates and some excited states at (almost) physical pion masses, but the most distinctive feature of excited states are decays. These are only captured for very few states in lattice QCD, as discussed later.

This talk consists of a number of lessons. In Sec. 2 I give an answer to the question: What is a resonance? I also discuss briefly one example how resonance positions in the complex energy plane look and stress the two-pole structure of the $\Lambda(1405)$. Then, in Sec. 3, I discuss the case of a well-separated resonance in a finite volume, which brings us in contact with the Lüscher equation. This is extended to the multi-channel case in Sec. 4. I show in particular how chiral symmetry is important for the extrapolation into the complex plane, which leads us to the two-pole structure of the $D_{0}^{\star}(2400)$ from the analysis of high-precision lattice QCD data on the coupled channel $D \pi, D \eta, D_{s} \bar{K}$ scattering with isospin $I=1 / 2$. I also point out that this two-pole structure is more common then so far believed. In Sec. 5, I discuss hadronic molecules, which are important players in the hadron spectrum. I address in particular the issues how to distinguish them from compact multi-quark states and the calculation of the production cross section in high-energy proton-(anti-)proton collisions. Then, in Sec. 6 I turn to the calculation of the width of the two lowest-lying baryon resonances, the $\Delta(1232)$ and the Roper $N^{*}(1440)$ using covariant baryon chiral perturbation theory and the complex-mass scheme. Sec. 7 contains a few remarks on the pion cloud of the nucleon and other hadrons, also often encountered at this workshop. I end with a short summary in terms of takehome messages.

\section{Lesson 1: What is a resonance?}

In the old times, resonances were searched for by looking at bumps in scattering cross sections. But as Moorhouse stated so eloquently: "Not every bump is a resonance and not every resonance is a bump" [1]. Now we know that resonances have complex properties, like their mass and

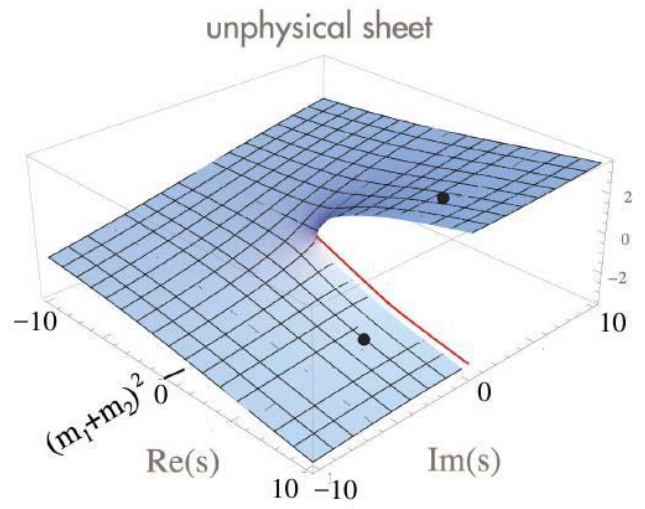

Figure 1. The imaginary part of a single-channel amplitude in the presence of a resonance. The solid dots indicate the allowed positions for resonance poles. Figure from [2].

width, their photo-couplings, etc. . In particular, these intrinsic properties do not depend on the experiment or theory (model). Most importantly, resonances correspond to S-matrix poles on unphysical Riemann sheets, as depicted in Fig. 1.

This is the most basic and the only acceptable definition of a resonance (with very few exceptions of wellisolated, single channel cases). So if somebody supplies you with the resonances parameters and has not looked for the corresponding pole in the complex plane, these numbers should be considered with suspicion or even be discarded. Especially in cases when a number of coupled channels is involved, the search in the complex energy plane is essentially the only viable method. A nice example of such a search in the multiple channel case (here: $\pi^{0} p, \pi^{+} n, p \eta, \Lambda K^{+}, \Sigma^{0} K^{+}$and $\Sigma^{+} K^{0}$ ) are the two close-by poles corresponding to the two lowest $S_{11}$ resonances in elastic pion-nucleon scattering with $J^{P}=1 / 2^{-}$as shown in Fig. 2 (from Ref. [3]). Another beautiful example is given by the two-pole structure of the $\Lambda(1405)$, first noted in Ref. [4]. Here, multiple channels need to be considered, namely $K^{-} p \rightarrow$ $K^{-} p, \bar{K}^{0} n, \Sigma^{0} \pi^{0}, \Sigma^{+} \pi^{-}, \Sigma^{-} \pi^{+}, \Lambda \pi^{0}, \Lambda \eta, \Xi^{+} K^{-}, \Xi^{0} K^{0}$. Only if one analyzes the pole structure in the complex energy plane, one finds these two poles, one being close to the $K^{-} p$ and the other closer to the $\pi \Sigma$ threshold, see Fig. 3. In fact, this two-pole structure has been verified by various groups world-wide, for a comparative study see [5] and later discussions.

Let us now consider the lattice, which corresponds to a finite, cubic box (keeping the time coordinate continuous for the moment). As it is well-known from quantum mechanics, the eigenstates of any Hamiltonian in a box are discrete energy levels. So does that mean that the excited states of QCD are not amenable to LQCD? Fortunately, as pointed out by Lüscher [6] and others, one can relate the volume dependence of the energy spectrum on the lattice to the continuum scattering phase shift. In fact, an isolated narrow resonance can be traced back to an avoided level crossing, see e.g. Ref. [7]. This, however, is not a practical method, so in the following sections I will discuss how to proceed in LQCD. 


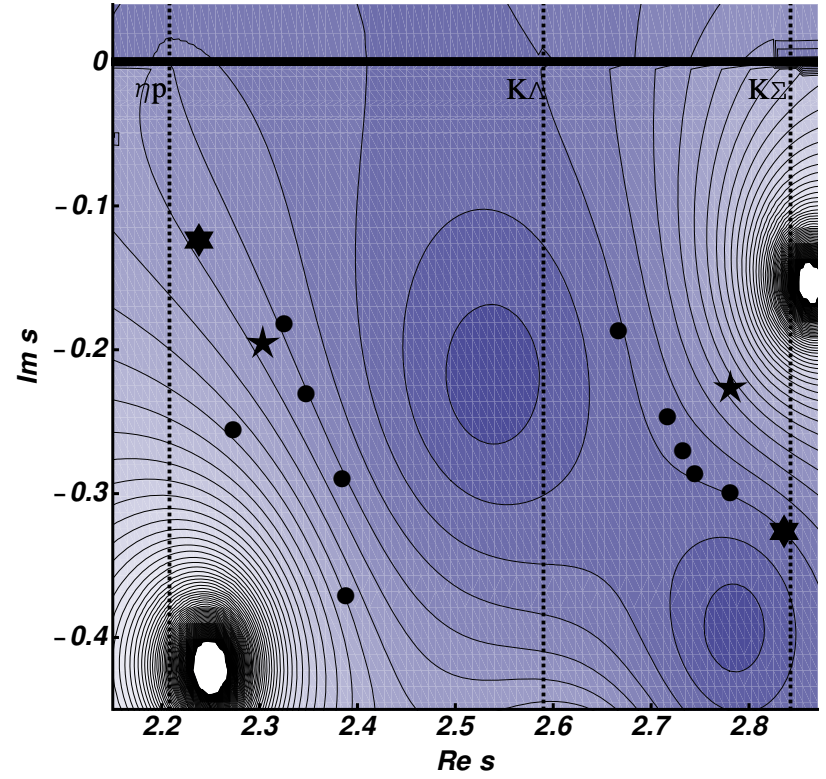

Figure 2. Modulus of the analytic continuation of the $J^{P}=1 / 2^{-}$ $\pi N$ scattering amplitude into the complex $s$-plane. Shown is the $(--+++)$ Riemann sheet. The two poles at $\sqrt{s}=$ $(1.51-i 0.14) \mathrm{GeV}$ and $\sqrt{s}=(1.69-i 0.05) \mathrm{GeV}$, corresponding to the $S_{11}(1535)$ and the $S_{11}(1650)$, respectively, are clearly visible. The stars and black dots refer to other determinations as listed in [3]. For the definitions of the sheets, see e.g. Ref. [5].

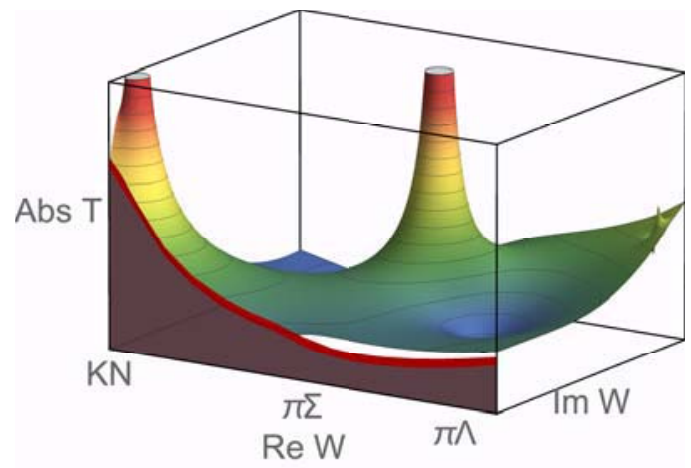

Figure 3. The S-wave amplitude $f_{0+}$ for $K^{-} p \rightarrow K^{-} p$ for $I=0$ in the complex energy plane clearly showing the two poles in the region of the $\Lambda(1405)$. The red band indicates that the $(++--$ --++++ ) Riemann sheet is only connected to the real energy axis between the $\bar{K} N$ and the $\pi \Sigma$ thresholds. For the definitions of the sheets, see e.g. Ref. [5]. Figure courtesy of Maxim Mai.

\section{Lesson 2: Well separated resonances}

Well separated resonances are, as already stated, more the exception than the rule. Still, they provide important benchmarks for any calculation of the spectrum. Let us consider an isolated resonance in a box. Before discussing the Lüscher formalism in three space dimensions, let me elaborate on the one-dimensional case as an instructive example. Consider the scattering of two particles on a line segment of length $L$ (the lattice size), with a finite interaction range $R \ll L$. For simplicity, assume further an infinite time extent. As expected, outside the range of the interaction, the relative motion of the scattering particles should be described by a plane wave. Then, the interaction produces a finite phase shift $\delta(k)$ as known from elementary scattering theory. Let us impose periodic boundary conditions in the spatial dimension. Hence, for a plane wave with momentum $k$, one has

$$
\exp (i k L+2 i \delta(k))=\exp (i k 0)=1
$$

which gives

$$
k_{n} L+2 \delta\left(k_{n}\right)=2 \pi n, \quad n \in \mathbb{N},
$$

for the quantization of the momenta. This result is remarkable, as it relates energies computed on the lattice to the continuum phase shift. Given an appropriate (lattice or continuum) dispersion relation which relates the lattice energy levels $E_{n}(L)$ to the momentum modes $k_{n}(L)$, the continuum phase shift at discrete values of the scattering momentum can be reconstructed from the energy levels on the lattice. Also, the non-interacting limit $\delta\left(k_{n}\right)=0$ is recovered for $k_{n}=2 \pi n / L$. For non-relativistic particles with mass $m$, this method is applicable when $m L \gg 1$, so that further corrections are exponentially suppressed. Also, any inelasticity or coupling to other scattering channels introduces modifications. Let me now discuss the case of three space dimensions. The energy levels for two noninteracting identical particles of mass $m$ are given by

$$
E_{n}(L)=2 \sqrt{m^{2}+\vec{k}^{2}}, \quad k_{i}=\frac{2 \pi}{L} n_{i}, n_{i} \in \mathbb{Z} .
$$

Turning on the interaction, we obtain the continuum scattering phase $\delta(k)$ with the help of Lüscher's formula,

$$
\begin{aligned}
\delta(k) & =-\phi(q) \bmod \pi, q=\frac{k L}{2 \pi}, \\
\phi(q) & =-\arctan \frac{\pi^{3 / 2} q}{\mathcal{Z}_{00}\left(1 ; q^{2}\right)}, \\
\mathcal{Z}_{00}\left(1 ; q^{2}\right) & =\frac{1}{\sqrt{4 \pi}} \sum_{\vec{n} \in \mathbb{Z}^{3}} \frac{1}{\vec{n}^{2}-q^{2}},
\end{aligned}
$$

for the S-wave. Note that the Lüscher $\zeta$-function $\mathcal{Z}_{00}$ requires regularization, which can be done in different ways (a specific example is given in the next section). Generalizations to higher partial waves also exist. If we assume a resonance with mass $m_{R}>2 m$, we can also use the effective range expansion (ERE),

$$
\tan \left(\delta-\frac{\pi}{2}\right)=\frac{E^{2}-m_{R}^{2}}{m_{R} \Gamma_{R}},
$$

with $m_{R}\left(\Gamma_{R}\right)$ the mass (width) of the resonance. Note, however, that this can only be used under very special circumstances (well isolated resonance, weak energy dependence of the background). In such a case, one can measure the phase shift in the resonance region and fit $m_{R}, \Gamma_{R}$, making also use of moving frames as pioneered in Ref. [8]. As an example, I show in Fig. 4 some pioneering (but unpublished) results for the $\Delta(1232)$, which is a well separated baryon resonance in the $\pi N$ system, corresponding to the $l=1, I=3 / 2 \pi N$ phase shift. These results were obtained for pion masses from 160 to $390 \mathrm{MeV}$ in fairly large volumes [9]. More precisely, the calculation 


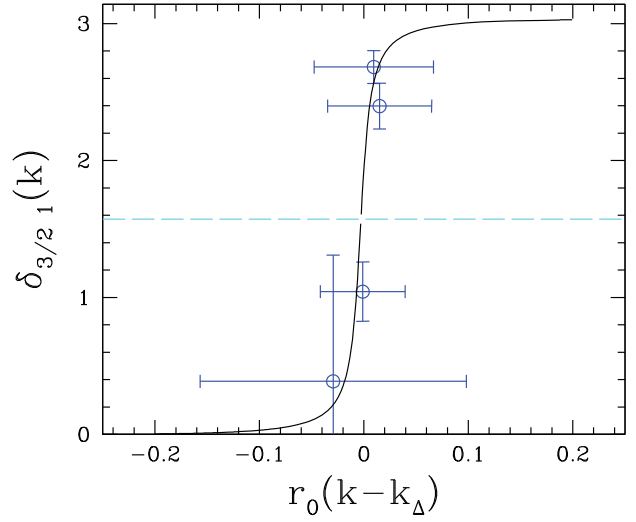

Figure 4. The $\pi N$ phase shift of the $\Delta$ channel as a function of the center-of-mass momentum $k$. The solid line is the phase generated from the physical mass and width of the $\Delta$-resonance. The dashed horizontal line indicates the $90^{\circ}$ crossing of the phase.

used a $N_{f}=2$ clover action that consists of the plaquette gluon action together with nonperturbatively $O(a)$ improved Wilson (clover) fermions on $32^{3} \times 64$ and $40^{3} \times 64$ lattices at $\beta=5.29, \kappa=0.13632, a=0.075 \mathrm{fm}$ and on $40^{3} \times 64$ and $48^{3} \times 64$ lattices at $\beta=5.29, \kappa=0.1364, a=$ $0.06 \mathrm{fm}$. More recent results on the $\Delta$ are given in the talks by Morningstar [10] and by Petschlies [11]. For single channel calculations of elastic pion-nucleon scattering in the $J^{P}=1 / 2^{-}$channel, see Refs. [12, 13], and for the Roper in the $J^{P}=1 / 2^{+}$channel, see e.g. Ref. [14].

In the meson sector, the genuine case of an isolated resonance is the $\rho$ that shows up in the $I=J=1$ channel of pion-pion scattering. Here, the most recent calculation is due to the ETM Collaboration [15], and I refer to that paper for details.

\section{Lesson 3: Coupled channels/thresholds}

As said before, isolated (well-separated) resonances are the exception, in most cases we have to deal with coupled channel effects and/or close-by thresholds, e.g. for the scalar mesons $f_{0}(980)$ and $a_{0}(980)$ or the two poles in the $\Lambda(1405)$ region. There exist various extensions of Lüscher's approach to cope with coupled channels: 1) a purely quantum mechanical treatment, see e.g. [16], 2) the formulation within non-relativistic EFT (NREFT), see e.g. [17-20], 3) the use of finite-volume unitarized CHPT, see e.g. [21, 22] or 4) a relativistic version, see e.g. [23]. The main advantage of the third method is that it is based on an effective chiral Lagrangian that allows one to relate various processes. This will be exemplified below. So far, these methods have been mostly applied in the meson sector, with a few exceptions also for baryons $[24,25]$. Before elaborating on one concrete example, I would like to issue a warning: Be aware of methods that can mislead you (K-matrix and alike). Some possible pitfalls will also be discussed in what follows.

The Hadron Spectrum Collaboration (HSC) has investigated the coupled channel $D \pi, D \eta, D_{s} \bar{K}$ scattering with isospin $I=1 / 2$ [26] (for S-, P- and D-waves). They presented results for three lattice volumes, one spatial lattice

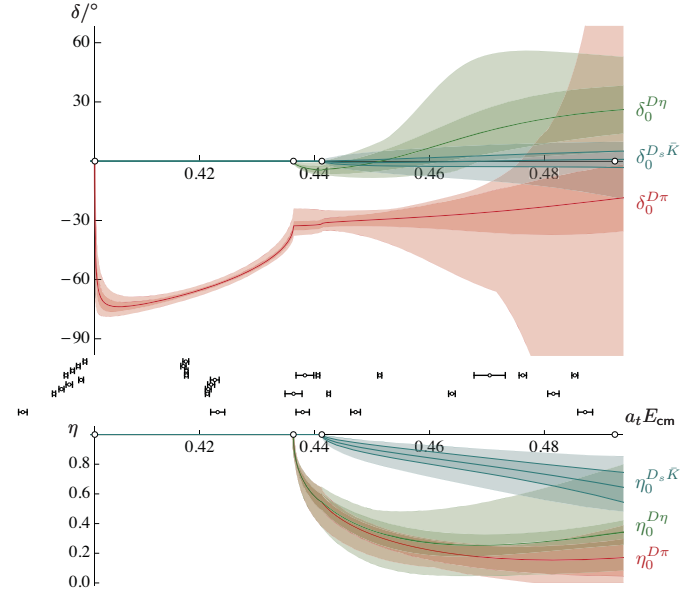

Figure 5. The upper (lower) panel shows the S-wave phase shifts (inelasticities) for the $D \pi$ (red), $D \eta$ (green) and $D_{s} \bar{K}$ (blue) channels, where the size of the bands incorporates all T-matrix parametrizations of the S-wave. The black points show the location of the finite-volume energy levels used to constrain the parametrizations. Figure from [26].

spacing $a_{s}$ and one temporal spacing $a_{t}$ at $M_{\pi} \simeq 390 \mathrm{MeV}$. Furthermore, they used various K-matrix type extrapolations (in fact, up to 11 variants) to look for poles in the complex plane. The resulting phase shifts and inelasticities are shown in Fig. 5. They report one S-wave pole at $(2275.9 \pm 0.9) \mathrm{MeV}$ that is located very closely to the $D \pi$ threshold. The authors of Ref. [26] argue that this pole shares similarities with the $D_{0}^{\star}(2400)$ of the Particle Data Group (PDG) [27] (note that the $D_{0}^{*}(2400)$ has been renamed as $D_{0}^{*}(2300)$ in the 2019 PDG update). However, in their T-matrix parametrizations, they ignored the important role of chiral symmetry. It is long known how to incorporate chiral symmetry in coupled-channel dynamics in the framework of unitarized CHPT (UCHPT), see e.g. the groundbreaking works in Refs. [4, 28-30]. To make contact to the work of the Hadron Spectrum Collaboration, let us consider the case of Goldstone bosons scattering off $D$-mesons ( $D \phi$ scattering). The corresponding T-matrix in UCHPT takes the form

$$
T^{-1}(s)=V^{-1}(s)-G(s) .
$$

Here, the potential $V(s)$ can be obtained from the SU(3) heavy-light chiral effective Lagrangian, which up to nextto-leading order (NLO) contains 6 low-energy constants (LECs), as discussed below. Further, $G(s)$ is the 2-point scalar loop function, which is best written in a dispersiontheoretical representation and regularized with a subtraction constant $a(\mu)$, see e.g. [4]. Of course, $T, V$ and $G$ are matrices in the channel space, but for clarity I have suppressed the channel indices. Let us now discuss the NLO effective chiral Lagrangian for $D \phi$ coupled channel dynamics. Following Ref. [31], it takes the form

$$
\begin{aligned}
& \mathcal{L}_{\text {eff }}=\mathcal{L}^{(1)}+\mathcal{L}^{(2)}, \\
& \mathcal{L}^{(1)}=\mathcal{D}_{\mu} D \mathcal{D}^{\mu} D^{\dagger}-M_{D}^{2} D D^{\dagger}
\end{aligned}
$$




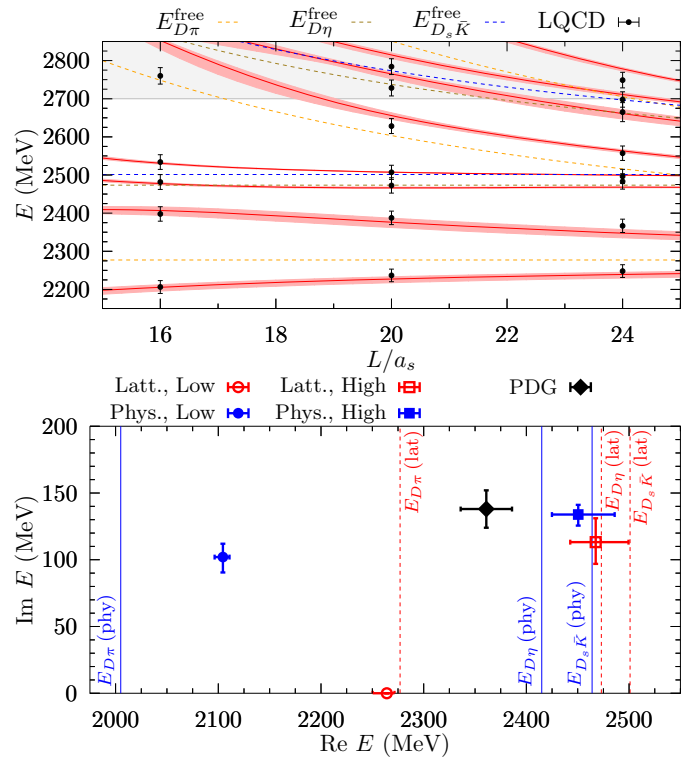

Figure 6. The upper panel shows the energy levels calculated in UCHPT with LECs determined in 2013 in comparison to the results of [26] in the $(S, I)=(0,1 / 2)$ channel. Lower panel: Complex energy plane location of the two-pole-structure. Empty red (filled blue) symbols stand for the poles obtained when the LQCD [26] (physical) masses are used. The black diamond represents the isospin average of the PDG values for $D_{0}^{\star}(2400)^{0}$ and $D_{0}^{\star}(2400)^{+}$. Figures from [33].

$$
\begin{aligned}
\mathcal{L}^{(2)} & =D\left[-h_{0}\left\langle\chi_{+}\right\rangle-h_{1} \chi_{+}\right. \\
& \left.+h_{2}\left\langle u_{\mu} u^{\mu}\right\rangle-h_{3} u_{\mu} u^{\mu}\right] D^{\dagger} \\
& +\mathcal{D}_{\mu} D\left[h_{4}\left\langle u^{\mu} u^{\nu}\right\rangle-h_{5}\left\{u^{\mu}, u^{\nu}\right\}\right] \mathcal{D}_{\nu} D^{\dagger},
\end{aligned}
$$

with $D=\left(D^{0}, D^{+}, D_{s}^{+}\right), \mathcal{D}_{\mu}$ is the chiral covariant derivative, $M_{D}$ the $D$-meson mass (in the chiral limit) and the conventional chiral building blocks $u_{\mu} \sim \partial_{\mu} \phi, \chi_{+} \sim \mathcal{M}$, etc. are used. The LECs can be determined as follows: $h_{0}$ can be fixed from the pion-mass dependence of the $D$ and $D_{s}$ masses and $h_{1}=0.42$ is given by the $D_{s}-D$ splitting. Further, $h_{2,3,4,5}$ are obtained from a fit to lattice data $(D \pi \rightarrow D \pi, D \bar{K} \rightarrow D \bar{K}, \ldots)$, see Ref. [32]. In that paper, 5 "simple" channels that do not contain disconnected diagrams where fitted and then UCHPT was used to predict the mass of the charm-strange scalar meson (the pole in the $(S, I)=(1,0)$ channel $)$ at $2315_{-28}^{+18} \mathrm{MeV}$, consistent with the PDG value for the $D_{s 0}^{\star}(2317)$. To make contact to the results from [26], we need the appropriate formulation of UCHPT on a finite volume (FV). As noted before, in that case the momenta get quantized $\sim 1 / L$ and the loop function $G(s)$ gets modified. Using the formalism of Ref. [21], we have (note the aforementioned regularization of the Lüscher function)

$$
\tilde{G}(s, L)=\lim _{\Lambda \rightarrow \infty}\left[\frac{1}{L^{3}} \sum_{\vec{n}}^{|\vec{q}|<\Lambda} I(\vec{q})-\int_{0}^{\Lambda} \frac{q^{2} d q}{2 \pi^{2}} I(\vec{q})\right],
$$

where $I(\vec{q})$ is the integrand of $G(s)$. The FV energy levels are obtained from the poles of $\tilde{T}(s, L)$ via $\tilde{T}^{-1}(s, L)=$ $V^{-1}(s)-\tilde{G}(s, L)$. Note that on a torus the potential $V(s)$ is the same as in the continuum. Using this framework, the HSC data were re-analyzed in Ref. [33]. First, the energy

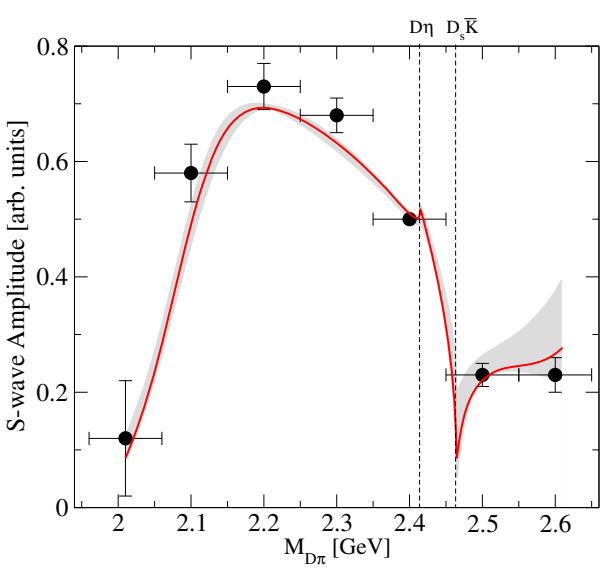

Figure 7. Comparison of the S-wave amplitude determined from the calculation of [39] with the S-wave anchor points from the experimental analysis, shown as the data points [42]. The red (solid) line gives the best fit results and the grey band quantifies the uncertainties. The dashed perpendicular lines indicate the location of the $D \eta$ and $D_{s} \bar{K}$ threshold, respectively. Figure from [39].

levels were postdicted (no parameter needed to be fixed) to a high precision as shown in the upper panel of Fig. 6 . Second, and even more stunning, was the observation of the two-pole structure of the $D_{0}^{\star}(2400)$, as depicted in the lower panel of Fig. 6, in complete analogy to the case of the $\Lambda(1405)$ [4]. This solves the enigma that the mass of the $D_{0}^{\star}(2400)$, which is made of a light $(u, d)$ and a charm quark, is larger than the one of the $D_{s 0}^{\star}(2317)$, which contains the heavier strange quark. The lower of the two poles is lying visibly below the $D_{s 0}^{\star}(2317)$ ! This two-pole structure is easily understood from group theory. Consider the SU(3) limit, where all light and heavy mesons take common values, analogous to the study of the $\Lambda(1405)$ in [34]. Combining the $D$-meson anti-triplet with the Goldstone boson octet gives

$$
\overline{3} \otimes 8=\underbrace{\overline{3} \oplus 6}_{\text {attractive }} \oplus \overline{15},
$$

where the anti-triplet and the sextet are attractive, leading to two zero-width poles. Once the SU(3) breaking in the meson masses is switched on, these poles move to the positions in the complex energy plane shown in Fig. 6 . We note that this two-pole structure had been observed in earlier calculations [35-38] but did not receive the proper attention then. In fact, as pointed out in Ref. [39], there is an easy lattice test for this scenario: The sextet pole becomes a bound state for $M_{\phi}>575 \mathrm{MeV}$ in the SU(3) limit. Such a calculation could be easily done and would be a fine test of the two-pole scenario. In fact, there is further phenomenological support of this picture. Analyzing the high-precision data on the decays $B \rightarrow D \phi \phi$ from $\mathrm{LHCb}$ [40-42] within the same framework (using the predetermined S-waves) leads to an excellent description of the so-called angular moments [39, 43] and a prediction of two cusps in the $\mathrm{S}$-wave at the $D \eta$ and $D_{s} \bar{K}$ thresholds, as shown in Fig. 7. Shown are the so-called anchor points provided by $\mathrm{LHCb}$, where the strength and the phase of 
Table 1. Predictions of two states in various $I=1 / 2$ channels in the heavy meson sector. Here $(M, \Gamma / 2)$ denote the mass and the half-width, respectively, in units of [MeV]. From Ref. [39]

\begin{tabular}{|c|cc|}
\hline & Lower pole & Higher pole \\
\hline$D_{0}^{\star}$ & $\left(2105_{-8}^{+6}, 102_{-11}^{+10}\right)$ & $\left(2451_{-26}^{+36}, 134_{-8}^{+7}\right)$ \\
$D_{1}$ & $\left(2247_{-6}^{+5}, 107_{-10}^{+11}\right)$ & $\left(2555_{-30}^{+47}, 203_{-9}^{+8}\right)$ \\
$B_{0}^{\star}$ & $\left(5535_{-11}^{+9}, 113_{-17}^{+15}\right)$ & $\left(5852_{-19}^{+16}, 36 \pm 5\right)$ \\
$B_{1}$ & $\left(5584_{-11}^{+9}, 119_{-17}^{+14}\right)$ & $\left(5912_{-18}^{+15}, 42_{-4}^{+5}\right)$ \\
\hline
\end{tabular}

the S-wave were extracted from the data and connected by a cubic spline in comparison to the UCHPT result of Ref. [39]. I point out that the higher mass pole at $2.45 \mathrm{GeV}$ amplifies the predicted cusps. In fact, the two-pole scenario can be extended to the axial-vector states and the corresponding $B$-mesons using HQSS and HQFS, respectively. The emerging picture based on the calculations in Ref. [39] (and references therein) is summarized in Table 1 . Note that the PDG lists one state for the $D_{0}^{\star}$ and for the $D_{1}$, these are located at $(2318 \pm 29,134 \pm 20) \mathrm{MeV}$ and $\left(2427 \pm 40,192_{-55}^{+65}\right) \mathrm{MeV}$, respectively. In view of the results obtained in UCHPT, time is ripe to change these entries in the PDG. The predicted two-pole scenarios for the $B_{0}^{\star}$ and the $B_{1}$ are a nice test to further validate this picture.

\section{Lesson 4: Hadronic molecules}

As already stated in the introduction, QCD offers yet another set of bound states, first seen in nuclear physics, namely hadronic molecules, which are bound states made of 2 or 3 hadrons. In what follows, I consider bound states of two hadrons in S-wave very close to a 2-particle threshold or between two close-by thresholds. Such molecular states are weakly bound, i.e. the binding energies are much smaller than the particle mass, and this weak binding also entails a large spatial extent. Further, hadronic molecules show particular decay patterns. The classical example is, of course, the deuteron, a bound state of a proton and a neutron. Its binding energy (BE) of about $2.22 \mathrm{MeV}$ is much smaller than its mass, $M_{D}=m_{p}+m_{n}-E_{B} \simeq$ $1876 \mathrm{MeV}$ and its radius of $2.14 \mathrm{fm}$ is much bigger than the proton radius of about $0.85 \mathrm{fm}$. Other examples are the two poles in the $\Lambda(1405)$ region, the $f_{0}(980)$, the $X(3872)$, and many others. For a recent review, see [2] and the discussion below.

Naturally the question arises how to distinguish these molecules from compact multi-quark states? This was originally answered by Weinberg [44] and then refined by various others, see e.g. [45-47]. For that, consider the wave function of a bound state $|\Psi\rangle$ with a compact component $\left|\psi_{0}\right\rangle$ and a two-hadron component $\left|h_{1} h_{2}\right\rangle$ in S-wave:

$$
|\Psi\rangle=\left(\begin{array}{c}
\sqrt{Z}\left|\psi_{0}\right\rangle \\
\chi(\vec{k})\left|h_{1} h_{2}\right\rangle
\end{array}\right),
$$

that is, such a bound state consists of a compact component with probability $\sqrt{Z}$ and a two-hadron component
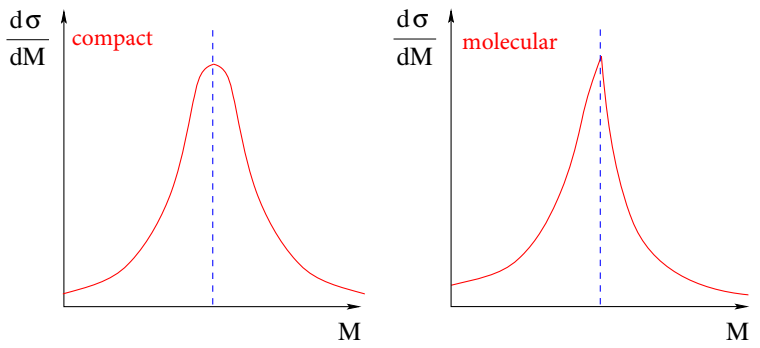

Figure 8. Typical near-threshold line shapes that emerge for compact (left panel) and molecular states (right panel). The dashed perpendicular lines indicate the location of the threshold. Note the cusp at the threshold for the molecular scenario. The $x$-axis shows $M=m_{1}+m_{2}+E$. Figure inspired from [2].

with a relative wave function $\chi(\vec{k})$ (which should be normalizable). Then, comparing the hadron-hadron scattering amplitude with the effective range expansion gives:

$$
\begin{gathered}
a=-2 \frac{1-Z}{2-Z}\left(\frac{1}{\gamma}\right)+O\left(\frac{1}{\beta}\right), \\
r=-\frac{Z}{1-Z}\left(\frac{1}{\gamma}\right)+O\left(\frac{1}{\beta}\right),
\end{gathered}
$$

with $\gamma=\sqrt{2 \mu E_{B}}$ the binding momentum, $\mu$ the reduced mass, $a$ the scattering length, $r$ the effective range and $\beta$ is the range of forces. For a pure molecule $(Z=0)$ we have the maximal scattering length, $a=-1 / \gamma$, and a natural effective range $r=O(1 / \beta)$. Very differently for a compact state $(Z=1)$, the scattering length $a=-O(1 / \beta)$ is of natural size and the effective range diverges, which is cured by higher order effects not explicitly displayed in Eq. (14). As an example, consider the deuteron. Here, we have $\gamma=45.7 \mathrm{MeV}=0.23 \mathrm{fm}^{-1}$ and the range of forces is set by the one-pion-exchange, $1 / \beta \sim 1 / M_{\pi} \simeq 1.4 \mathrm{fm}$. Setting $Z=0$ in Eq. (14), we find $a_{\mathrm{mol}}=-(4.3 \pm 1.4) \mathrm{fm}$ and $r_{\mathrm{mol}}=1.4 \mathrm{fm}$, consistent with the data, $a=-5.419(7) \mathrm{fm}$ and $r=1.764(8) \mathrm{fm}$.

Besides the deuteron all other (candidates for) hadronic molecules are unstable. Then the scattering Tmatrix needs to be modified compared to the form discussed before. The corresponding modifications of the framework to cope with hadron resonances have been developed, see e.g. Refs. [47-50]. Assuming again closeness to a two-particle threshold, the scattering amplitude takes the form

$$
\mathrm{T}(E)=\frac{g^{2} / 2}{E-E_{R}+\left(g^{2} / 2\right)(i k+\gamma)+i \Gamma_{0} / 2},
$$

with $g$ the coupling constant of the two-hadron system, $E=k^{2} /(2 \mu), E_{R}$ the resonance energy and $\Gamma_{0}$ accounts for the inelasticities of other channels. This leads to very different line shapes for compact and molecular states. While for a compact state the $k^{2}$ term dominates leading to a symmetric line shape, for a molecular state the $g^{2}$ term dominates, leading to an asymmetric distribution exhibiting a cusp, cf. Fig. 8. Further generalizations to instable constituents, virtual poles, etc., have also been worked out. I refer again to the review [2] for details. 


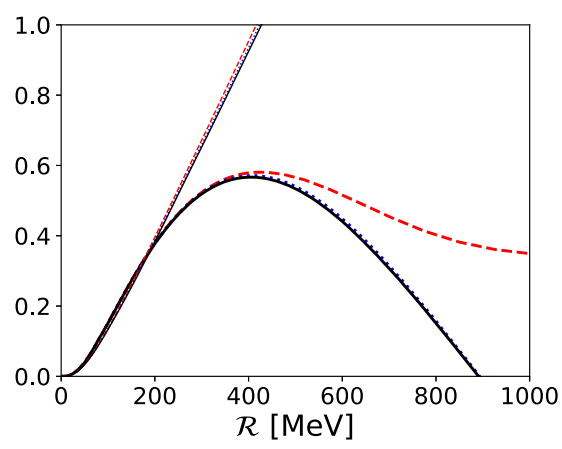

Figure 9. Estimate of $\bar{\Psi}_{\lambda}(\mathcal{R})$ for various deuteron wave functions: Results for $\lambda=0.8,1.6,4 \mathrm{GeV}$ are shown as red (dashed), blue (dotted) and black (solid) curves, respectively. The thick (thin) lines depict the results with (without) OPE. Figure from [63].

Without going into details, let me list a few candidates for hadronic molecules (for details, see e.g. Ref. [2]). Prominent examples in the light quark sector are the scalar mesons $f_{0}(980), a_{0}(980)$ as well as the already mentioned two $\Lambda(1405)$ states, here I refer to the talks by Mai [51] and Oset [52] for further discussion. There are also very prominent examples in the $c \bar{c}$ and $b \bar{b}$ spectrum, these are the $X(3872), Z_{c}(3900), Y(4260), Y(4660)$, $Z_{b}(10610), Z_{b}(10650)$, and others. Similarly, in heavylight systems we have a number of candidates, especially the $D_{s 0}^{\star}(2317), D_{s 1}(2460), D_{s 1}^{\star}(2860)$, and others. The molecular nature of the charm-strange mesons was already discussed in Sect. 4. In the baryon sector, the LHCb pentaquarks $[53,54]$ are good candidates, especially the recently observed $P_{c}(4457)$, see e.g. Refs. [55-61].

I will take the opportunity to discuss a few misconceptions surrounding hadronic molecules. It is often claimed that molecules due to their large spatial extent can not be produced in high-energy collisions, say at the LHC, but this is not correct. An argument found in the literature is due to Ref. [62]. They consider the $X(3872)$ production at the Tevatron, and derived a bound on the cross section:

$$
\begin{aligned}
\sigma(\bar{p} p \rightarrow X) & \sim\left|\int^{3} d^{3}\left\langle X \mid D^{0} \bar{D}^{* 0}(\mathbf{k})\right\rangle\left\langle D^{0} \bar{D}^{* 0}(\mathbf{k}) \mid \bar{p} p\right\rangle\right|^{2} \\
& \simeq\left|\int_{\mathcal{R}} d^{3} \mathbf{k}\left\langle X \mid D^{0} \bar{D}^{* 0}(\mathbf{k})\right\rangle\left\langle D^{0} \bar{D}^{* 0}(\mathbf{k}) \mid \bar{p} p\right\rangle\right|^{2} \\
& \leq \int_{\mathcal{R}} d^{3} \mathbf{k}|\Psi(\mathbf{k})|^{2} \int_{\mathcal{R}} d^{3} \mathbf{k}\left|\left\langle D^{0} \bar{D}^{* 0}(\mathbf{k}) \mid \bar{p} p\right\rangle\right|^{2} \\
& \leq \int_{\mathcal{R}} d^{3} \mathbf{k}\left|\left\langle D^{0} \bar{D}^{* 0}(\mathbf{k}) \mid \bar{p} p\right\rangle\right|^{2}
\end{aligned}
$$

which depends crucially on the value of $\mathcal{R}$ which specifies the region where the bound state wave function " $\Psi(\mathbf{k})$ is significantly different from zero" [62]. These authors assume that $\mathcal{R}$ is of the order of the binding momentum $\gamma$, which amounts to $\mathcal{R} \simeq 35 \mathrm{MeV}$ for the then accepted $\mathrm{BE}$ of the $X(3872)$. From this they conclude that $\sigma(\bar{p} p \rightarrow X) \simeq 0.07 \mathrm{nb}$, which is orders of magnitudes lower than the experimental bounds known then (updated numbers will be given below). So this leads them to con-

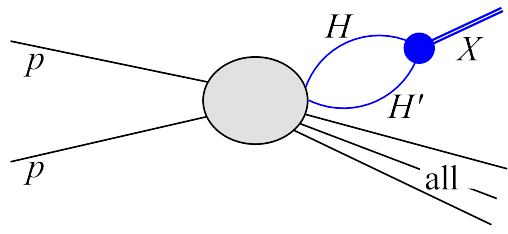

Figure 10. The mechanism for the inclusive production of the $X$ as a $H H^{\prime}$ bound state in proton-proton collisions. Here, all denotes all the produced particles other than the $H$ and $H^{\prime}$ in the collision. Figure from [66].

clude that the $X(3872)$ can not be a molecule. But I said that the $X(3872)$ is a premier candidate for a molecule in the charmonium spectrum, so what goes wrong with this argument? Let us take a step back and consider the best known molecule, the deuteron [63]. The relevant integral is

$$
\bar{\Psi}_{\lambda}(\mathcal{R}) \equiv \int_{\mathcal{R}} d^{3} \mathbf{k} \Psi_{\lambda}(\mathbf{k}),
$$

where $\lambda$ specifies a regulator that needs to be introduced to render the wave function well defined. In the deuteron case, $\gamma \simeq 45 \mathrm{MeV}$, and as in the case of the $X(3872)$ is of the order of the pion Compton wavelength $O\left(1 / M_{\pi}\right)$. In Fig. 9, $\bar{\Psi}_{\lambda}(\mathcal{R})$ is shown for the deuteron with two sets of wave functions, one generated from a potential with a short-ranged term and one-pion exchange (OPE) and the other without OPE. For the former a Gaussian regulator is used and the small $D$-wave component is not shown. For the latter a sharp momentum space cut off is used for simplicity. One sees that $\bar{\Psi}_{\lambda}(\mathcal{R})$ is far from being saturated for $\mathcal{R} \simeq \gamma_{d}$ for all values of $\lambda$. A much larger value of $\mathcal{R} \sim 300 \mathrm{MeV} \sim 2 M_{\pi}$ needs to be taken for the second line in Eq. (16) to be a good approximation of the first. This is, of course, related to the fact that two-pion exchange is required to bind the deuteron. The same should also be true for the $X(3872)$ as a $D \bar{D}^{*}$ molecule, since the range of forces is the same. A similar value was also favored in Ref. [64] based on rescattering arguments. In fact, Ref. [62] noted that with such a value of $\mathcal{R}$ the upper bound becomes consistent with the CDF result.

Let me briefly discuss how one can calculate the hadroproduction of the $X(3872)$ in an EFT if one realizes that this is a process involving short-distance physics, but still factorization is at work [64]. In such a scheme, event generators are used to calculate the collinear production of two hadrons and these then combine to form a molecule at large distances, see Fig. 10, which means that the molecule is not promptly produced. For high-energy processes like at the Tevatron or the LHC, the corresponding cross section of producing a molecule $X$ from two hadrons $H, H^{\prime}$ can be calculated via $[65,66]$ :

$$
\begin{gathered}
\sigma[X]=\frac{1}{4 m_{H} m_{H^{\prime}}} g^{2}|G|^{2}\left(\frac{d \sigma\left[H H^{\prime}(k)\right]}{d k}\right)_{\mathrm{MC}} \frac{4 \pi^{2} \mu}{k^{2}}, \\
G(E, \Lambda)=-\frac{\mu}{\pi^{2}}\left[\sqrt{2 \pi} \frac{\Lambda}{4}+\sqrt{\pi} \gamma D\left(\frac{\sqrt{2} \gamma}{\Lambda}\right)\right. \\
\left.-\frac{\pi}{2} \gamma e^{2 \gamma^{2} / \Lambda^{2}}\right],
\end{gathered}
$$


Table 2. Integrated cross sections (in units of nb) for $p p / \bar{p} \rightarrow X(3872)$ compared to experimental measurements by CDF [69] and CMS [70].

\begin{tabular}{|c|cr|}
\hline$\sigma(p p / \bar{p}) \rightarrow X(3872))$ & $\Lambda \in[0.5,1.0] \mathrm{GeV}$ & Exp. \\
\hline Tevatron & $5-29[\mathrm{nb}]$ & $37-115[\mathrm{nb}]$ \\
LHC7 & $4-55[\mathrm{nb}]$ & $13-39[\mathrm{nb}]$ \\
\hline
\end{tabular}

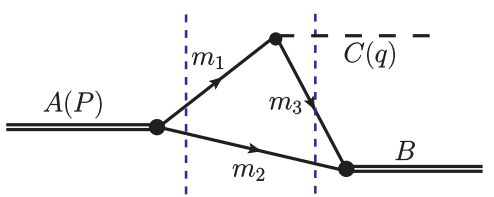

Figure 11. A triangle diagram illustrating the long-distance contribution to the transition between two heavy particles $\mathrm{A}$ and $\mathrm{B}$ with the emission of a light particle $\mathrm{C}$. The two vertical dashed lines denote the two relevant cuts. Figure from [2].

with $\left(d \sigma\left[H H^{\prime}(k)\right] / d k\right)_{\mathrm{MC}}$ the cross section of producing the hadrons $H$ and $H^{\prime}$ in collinear geometry, $G$ is the cutoff regularized two-meson $\left(H H^{\prime}\right)$ loop function expressed in terms of the Dawson function, $D(x)=e^{x^{2}} \int_{0}^{x} e^{-y^{2}} d y$, $\gamma=\sqrt{-2 \mu\left(E-m_{H}-m_{H^{\prime}}\right)}$ is the binding momentum and $\Lambda$ is the cutoff. Following Ref. [67], a range of $[0.5,1.0] \mathrm{GeV}$ is used for the cutoff $\Lambda$. Typical results using the PYTHIA and HERWIG generators are collected in Tab. 2. We see that the calculations are not very precise, but they are perfectly consistent with the data, very different from the conclusions drawn in Ref. [62]. For a similar calculation concerning the hadroproduction of the charm-strange mesons, I refer to Ref. [68].

Finally, I mention that most candidates for hadronic molecules have been found through decays that often involve triangle diagrams with an anomalous singularity, as studied by Landau, Nambu and others in the 1950ties. There exist different EFTs that are used in the calculations of hadronic molecules and their decays. These are the already mentioned UCHPT as well as $\mathrm{NREFT}_{1}$ and $\mathrm{NREFT}_{2}$ when at least one heavy quark is involved. In $\mathrm{NREFT}_{1}$, all intermediate particles in the triangle diagram in Fig. 11 are close to their mass shell, so that one can expand in powers of the average velocity and external (small) momenta. This has e.g. been applied to systematic studies of a number of charmonium transitions. In $\mathrm{NREFT}_{2}$ one intermediate particle is further off its mass shell, so one integrates out this particle and then proceeds as before. This EFT was originally invented as XEFT for studies of the $X(3872)$ [71]. XEFT resembles much the pionless EFT of nuclear physics, see e.g. [72]. It has been used for systematic studies of processes involving the $X(3872)$ and the $Z_{b}$ states, see e.g. [73].

\section{The width of the lightest nucleon resonances from baryon CHPT}

In this section, I will be concerned with calculating the width of the two lightest baryon resonances, the $\Delta(1232)$ and the Roper $N^{*}(1440)$. This might at first sight appear irritating, as imaginary parts are usually not precisely reproduced in CHPT. For that simple reason, one has to employ a complex-mass scheme and work to two loops. The complex-mass renormalization scheme is a method that was originally introduced for precision $W, Z$-physics, see e.g. [74, 75] and later transported to chiral EFT [76].

Let me give a brief outline of the complex-mass scheme (CMS), following Ref. [77]. Consider first an instable particle at tree level. The CMS amounts to treating the mass of this particle consistently as a complex quantity, defined as the location of the pole in the complex $k^{2}$ plane of the corresponding propagator with momentum $k$. It can be shown that this scheme is symmetry-preserving and leaves the corresponding Ward identities intact. Extending this to one loop, one splits the real bare masses into complex renormalized masses and complex counterterms. This is important, as only renormalized masses are observable. The corresponding Lagrangian yields Feynman rules with complex masses and counterterms, which allows for standard perturbative calculations. This is essentially a rearrangement of contributions that is not affected by double counting. The imaginary part of the particle mass appears in the propagator and is resummed in the Dyson series. In contrast to this, the imaginary part of the counterterm is not resummed. One can show that in such a case gauge invariance remains valid, and unitarity cancellations are respected order by order in the perturbative expansion. This also requires integrals with complex internal masses, as worked out in Ref. [78]. For further discussions of the method, the reader is referred to Ref. [77] (and references therein). In case of a chiral EFT, the perturbative expansion proceeds as usual in terms of small momenta and quark masses, with a proper treatment of the heavy particle mass in loop diagrams (like the heavybaryon scheme or the so-called infrared-regularization or the extended-on-mass-scheme discussed below).

\subsection{The width of the $\Delta(1232)$}

Consider first the width of the $\Delta$ at two-loop order [79]. The pertinent effective Lagrangian contains, besides many other terms, the leading $\pi \Delta$ and $\pi N \Delta$ couplings, parametrized in terms of the LECs $g_{1}$ and $h$, respectively,

$$
\begin{aligned}
\mathcal{L}_{\pi \Delta}^{(1)} & =-\bar{\Psi}_{\mu}^{i} \xi_{i j}^{\frac{3}{2}}\left\{\left(i D^{j k}-m_{\Delta} \delta^{j k}\right) g^{\mu \nu}\right. \\
& -i\left(\gamma^{\mu} D^{v, j k}+\gamma^{v} D^{\mu, j k}\right)+i \gamma^{\mu} D^{j k} \gamma^{v} \\
& +m_{\Delta} \delta^{j k} \gamma^{\mu} \gamma^{v}+g_{1} \frac{1}{2} l^{j k} \gamma_{5} g^{\mu v} \\
& +g_{2} \frac{1}{2}\left(\gamma^{\mu} u^{v, j k}+u^{v, j k} \gamma^{\mu}\right) \gamma_{5} \\
& \left.+g_{3} \frac{1}{2} \gamma^{\mu} \mu^{j k} \gamma_{5} \gamma^{v}\right) \xi_{k l}^{\frac{3}{2}} \Psi_{v}^{l}, \\
\mathcal{L}_{\pi N \Delta}^{(1)} & =h \bar{\Psi}_{\mu}^{i} \xi_{i j}^{\frac{3}{2}} \Theta^{\mu \alpha}\left(z_{1}\right) \omega_{\alpha}^{j} \Psi_{N}+\text { h.c. }, \\
\mathcal{L}_{\pi N \Delta}^{(2)} & =\bar{\Psi}_{\mu}^{i} \xi_{i j}^{\frac{3}{2}} \Theta^{\mu \alpha}\left(z_{2}\right) \\
& \times\left[i b_{3} \omega_{\alpha \beta}^{j} \gamma^{\beta}+i \frac{b_{8}}{m} \omega_{\alpha \beta}^{j} i D^{\beta}\right] \Psi_{N}+\text { h.c. },
\end{aligned}
$$




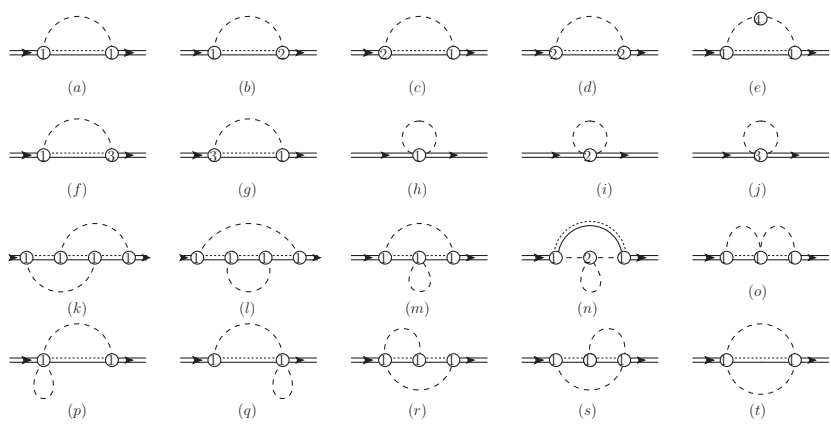

Figure 12. One and two-loop self-energy diagrams contributing to the width of the delta resonance up-to-and-including fifth order according to the standard power counting. The dashed and double solid lines represent the pions and the delta resonances, respectively. The double (solid-dotted) lines in the loops correspond to either nucleons or deltas. The numbers in the circles give the chiral orders of the vertices. Figure from [79].

$$
\begin{aligned}
\mathcal{L}_{\pi N \Delta}^{(3)} & =\bar{\Psi}_{\mu}^{i} \xi_{i j}^{\frac{3}{2}} \Theta^{\mu v}\left(z_{3}\right)\left[\frac{f_{1}}{m}\left[D_{v}, \omega_{\alpha \beta}^{j}\right] \gamma^{\alpha} i D^{\beta}\right. \\
& -\frac{f_{2}}{2 m^{2}}\left[D_{v}, \omega_{\alpha \beta}^{j}\right]\left\{D^{\alpha}, D^{\beta}\right\} \\
& \left.+f_{4} \omega_{\nu}^{j}\left\langle\chi_{+}\right\rangle+f_{5}\left[D_{v}, i \chi_{-}^{j}\right]\right] \Psi_{N}+\text { h.c. },
\end{aligned}
$$

where $\Psi_{N}$ and $\Psi_{v}$ are the isospin doublet field of the nucleon and the vector-spinor isovector-isospinor RaritaSchwinger field of the $\Delta$-resonance with bare masses $m$ and $m_{\Delta 0}$, respectively. $\xi^{\frac{3}{2}}$ is the isospin-3/2 projector, $\omega_{\alpha}^{i}=\frac{1}{2}\left\langle\tau^{i} u_{\alpha}\right\rangle$ and $\Theta^{\mu \alpha}(z)=g^{\mu \alpha}+z \gamma^{\mu} \gamma^{\alpha}$. Using field redefinitions the off-shell parameters $z$ can be absorbed in LECs of other terms of the effective Lagrangian and therefore they can be chosen arbitrarily $[80,81]$. We fix the offshell structure of the interactions with the delta by adopting $g_{2}=g_{3}=0$ and $z_{1}=z_{2}=z_{3}=0$. For vanishing external sources, the covariant derivatives are given by

$$
\begin{aligned}
D_{\mu} \Psi_{N} & =\left(\partial_{\mu}+\Gamma_{\mu}\right) \Psi_{N}, \\
\Gamma_{\mu} & =\frac{1}{2}\left[u^{\dagger} \partial_{\mu} u+u \partial_{\mu} u^{\dagger}\right]=\tau_{k} \Gamma_{\mu, k}, \\
\left(D_{\mu} \Psi\right)_{v, i} & =\partial_{\mu} \Psi_{v, i}-2 i \epsilon_{i j k} \Gamma_{\mu, k} \Psi_{v, j}+\Gamma_{\mu} \Psi_{v, i} .
\end{aligned}
$$

The power counting rests on $m_{\Delta}-m_{N}$ being a small quantity. More precisely, the small parameters are the external momenta, the pion mass and the nucleon-Delta mass splitting, collectively denoted as $q$. However, there are so many LECs in Eq. (19), so how can one one possibly make a prediction? Let us evaluate the $\Delta$ self-energy on the complex pole,

$$
z-m_{\Delta}^{0}-\Sigma(z)=0 \quad \text { with } \quad z=m_{\Delta}-i \frac{\Gamma_{\Delta}}{2} .
$$

The corresponding diagrams for the one- and two-loop self-energy contributing to the width of the delta resonance up to order $q^{5}$ are displayed in Fig. 12, where the counterterm diagrams are not shown. The one-loop diagrams are easily worked out. For the calculation of the two-loop graphs one uses the Cutkosky rules for instable particles, that relate the width to the pion-nucleon scattering amplitude, $\Gamma_{\Delta} \sim|A(\Delta \rightarrow N \pi)|^{2}$ [82]. One finds a remarkable

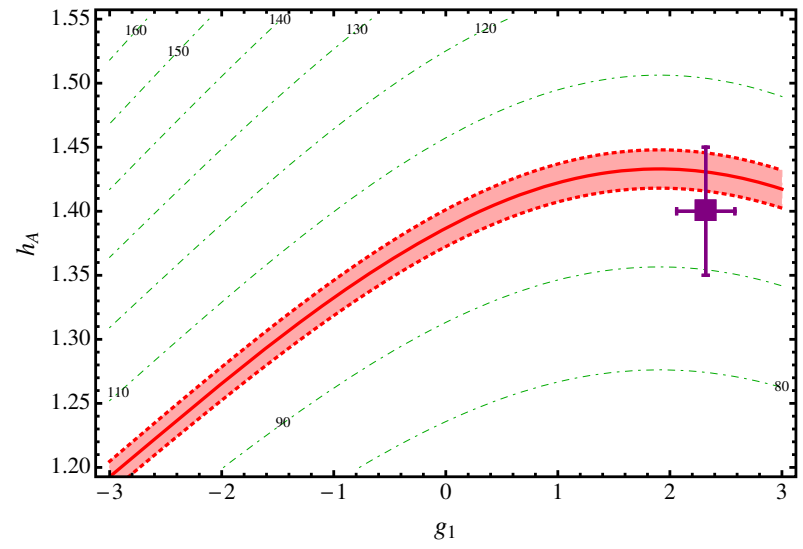

Figure 13. Correlation between the leading $\pi \Delta$ and $\pi N \Delta$ couplings. The central line corresponds to $\Gamma_{\Delta}=100 \mathrm{MeV}$ while the band is obtained by varying $\Gamma_{\Delta}$ in the range of 98 to $102 \mathrm{MeV}$. The dot-dashed lines show the correlation for other values of the width of the Delta. The box with the error bars are the results from the analysis of Ref. [84]. Figure inspired from [79].

reduction of parameters that is reflected in the relation

$$
\begin{aligned}
h_{A} & =h-\left(b_{3} \Delta_{23}+b_{8} \Delta_{123}\right) \\
& -\left(f_{1} \Delta_{23}+f_{2} \Delta_{123}\right) \Delta_{123}+2\left(2 f_{4}-f_{5}\right) M_{\pi}^{2} \\
\Delta_{23} & =m_{N}-m_{\Delta} \\
\Delta_{123} & =\frac{M_{\pi}^{2}+m_{N}^{2}-m_{\Delta}^{2}}{2 m_{N}}
\end{aligned}
$$

which means that all of the LECs appearing in the $\pi N \Delta$ interaction at second and third order, the $b_{i}(i=3,8)$ and $f_{i}(i=1,2,4,5)$, respectively, merely lead to a renormalization of the LO $\pi N \Delta$ coupling $h$, and, consequently, one finds a very simple formula for the decay width $\Delta \rightarrow N \pi$,

$\Gamma(\Delta \rightarrow N \pi)=\left(53.9 h_{A}^{2}+0.9 g_{1}^{2} h_{A}^{2}-3.3 g_{1} h_{A}^{2}-1.0 h_{A}^{4}\right) \mathrm{MeV}$.

This leads to a novel correlation that is independent of the number of colors, as $N_{c}$ was not used as a parameter in the calculation. This correlation between $h_{A}$ and $g_{1}$ is depicted in Fig. 13. It is obviously fulfilled by the analysis of Ref. [83], that showed that the inclusion of the $\Delta$ alleviates the tension between the threshold and subthreshold regions in the description of $\pi N$ scattering found in baryon CHPT.

\subsection{The width of the Roper resonance}

Next, I consider the calculation of the width of the Roper $N^{*}(1440)$ at two-loop order [85]. A remarkable feature of the Roper is the fact that its decay width into a nucleon and a pion is similar to the width into a nucleon and two pions. Any model that is supposed to describe the Roper must account for this fact. In CHPT, consider the effective chiral Lagrangian of pions, nucleons and deltas coupled to the Roper [86-88],

$$
\begin{aligned}
\mathcal{L}_{\mathrm{eff}} & =\mathcal{L}_{\pi \pi}+\mathcal{L}_{\pi N}+\mathcal{L}_{\pi \Delta}+\mathcal{L}_{\pi R} \\
& +\mathcal{L}_{\pi N \Delta}+\mathcal{L}_{\pi N R}+\mathcal{L}_{\pi \Delta R},
\end{aligned}
$$


with

$$
\begin{aligned}
& \mathcal{L}_{\pi R}^{(1)}=\bar{\Psi}_{R}\left\{i \not D-m_{R}+\frac{1}{2} g_{R} \psi l \gamma^{5}\right\} \Psi_{R}, \\
& \mathcal{L}_{\pi R}^{(2)}=\bar{\Psi}_{R}\left\{c_{1}^{R}\langle\chi+\rangle\right\} \Psi_{R}+\ldots, \\
& \mathcal{L}_{\pi N R}^{(1)}=\bar{\Psi}_{R}\left\{\frac{1}{2} g_{\pi N R} \gamma^{\mu} \gamma_{5} u_{\mu}\right\} \Psi_{N}+\text { h.c. }, \\
& \mathcal{L}_{\pi \Delta R}^{(1)}=h_{R} \bar{\Psi}_{\mu}^{i} \xi_{i j}^{\frac{3}{2}} \Theta^{\mu \alpha}(\tilde{z}) \omega_{\alpha}^{j} \Psi_{R}+\text { h.c. },
\end{aligned}
$$

where $g_{R}, g_{\pi N R}$ and $h_{R}$, respectively, are the leading Roper-pion, Roper-nucleon-pion and Delta-Roper-pion couplings. Here, $\Psi_{R}$ denotes the Roper isospin doublet field and all other notations are as in the preceeding subsection and in [85].

In this case, the power counting is more complicated, but can be set up around the complex pole as (for more details, see [85]), assigning the following counting rules:

$m_{R}-m_{N} \sim \varepsilon, m_{R}-m_{\Delta} \sim \varepsilon^{2}, m_{\Delta}-m_{N} \sim \varepsilon^{2}, M_{\pi} \sim \varepsilon^{2}$,

where $\varepsilon$ denotes a small parameter. Again, let us calculate the self-energy to two loops at the complex pole $z_{R}=m_{R}-$ $i \Gamma_{R} / 2$. By applying the cutting rules to these self-energy diagrams, one obtains the graphs contributing to the decay amplitudes of the Roper resonance into the $\pi N$ and $\pi \pi N$ systems, leading to the total width

$$
\Gamma_{R}=\Gamma_{R \rightarrow N \pi}+\Gamma_{R \rightarrow N \pi \pi} .
$$

A somewhat lengthy calculation leads to:

$$
\Gamma(R \rightarrow N \pi)=550(58) g_{\pi N R}^{2} \mathrm{MeV},
$$

and

$$
\begin{aligned}
\Gamma(R & \rightarrow N \pi \pi)=\left(1.5(0.6) g_{A}^{2} g_{\pi N R}^{2}\right. \\
& -2.8(1.0) g_{A} g_{\pi N R}^{2} g_{R} \\
& +1.5(0.6) g_{\pi N R}^{2} g_{R}^{2}+3.0(1.0) g_{A} g_{\pi N R} h_{A} h_{R} \\
& \left.-3.8(1.4) g_{\pi N R} g_{R} h_{A} h_{R}+9.9(5.5) h_{A}^{2} h_{R}^{2}\right) \mathrm{MeV} .
\end{aligned}
$$

The total width thus depends on five LECs. The uncertainties in the round brackets are generated by the uncertainties in the LECs. We use $g_{A}=1.27$ and $h_{A}=1.42 \pm 0.02$. The latter value is the real part of this coupling taken from Ref. [84]. As for the other unknown parameters, the authors of [85] fixed $g_{\pi N R}$ so as to reproduce the width $\Gamma_{R \rightarrow \pi N}=(123.5 \pm 19.0) \mathrm{MeV}$ from the PDG. This yields $g_{\pi N R}= \pm(0.47 \pm 0.04)$. In what follows, let us take the positive sign for our central value and use the negative one as part of the error budget. Further, assume $g_{R}=g_{A}$ and $h_{R}=h_{A}$, the so-called maximal mixing assumption [89]. Then, one can make a prediction for the two-pion decay width of the Roper,

$$
\Gamma(R \rightarrow N \pi \pi)=\left(41 \pm 22_{\mathrm{LECs}} \pm 17_{\text {h.o. }}\right) \mathrm{MeV},
$$

which is consistent with the PDG value of $(67 \pm 10) \mathrm{MeV}$. The error due to the neglect of the higher orders (h.o.) is simply given by multiplying the $\varepsilon^{5}$ result (central value) with $\varepsilon=\left(m_{R}-m_{N}\right) / m_{N} \simeq 0.43$. Clearly, to make further progress, we need an improved determination of the LECs $g_{R}$ and $h_{R}$. This could be addressed within LQCD. Finally, I would like to mention that this scheme has also been used to consider the electromagnetic transition form factors of the Roper [90].

\section{On the pion cloud of the nucleon and other hadrons}

At this conference, one often encountered the situation that some observable (like e.g. a resonance transition form factor extracted from pion electroproduction experiments) is well described in terms of quark degrees of freedom at large momentum transfer, say $Q^{2}$ a few $\mathrm{GeV}^{2}$, but then deviations between the model/theory show up when one approaches the photon point at $Q^{2}=0$. This is often cured by adding the pion cloud contribution from some hadronic model, see e.g. some examples in the opening talk by Burkert [91]. I would like to take the opportunity to issue a warning here. While the notion of the "pion cloud" is fairly intuitive, it is rather difficult to assign to it a quantitative measure. So let us look at that in more detail.

The so-called pion cloud is best analyzed in chiral perturbation theory. There is one very intriguing structural aspect generated by the (almost) massless Goldstone bosons of QCD, namely the so-called chiral logarithms $(\log s)$ or chiral singularities. Pions couple to themselves and to matter fields like the nucleons, thus generating a cloud that is Yukawa-suppressed at large distances as $\exp \left(-M_{\pi} r\right) / r$ for finite pion mass. In the chiral limit of vanishing quark and thus pion masses $M_{\pi} \rightarrow 0$, this Yukawa tail turns into a long-range Coulomb-like form. Consequently, S-matrix elements or transition currents can diverge in this limit. Famous examples are the pion vector radius or the nucleon isovector radius that scale as $\log \left(M_{\pi}\right)$, see e.g. Ref. [92], or the nucleon electromagnetic polarizabilities that are proportional to $1 / M_{\pi}$ [93]. This is not a disaster but rather a natural consequence of having massless degrees of freedom, and it can serve as an important check for other calculational schemes, like e.g. the lattice formulation of QCD. Also, any model that is supposed to describe the pion or the nucleon structure at low energies should obey such constraints. Note also that such singularities are generated by loop graphs, so that in general there will also be a pion-mass-independent contribution from a counterterm (contact interaction) at the same order of the calculation. A typical example is the isovector charge radius of the pion, or any other observable that displays a chiral log. Since the argument of a log must be a number, it really depends on $M_{\pi} / \mu$, with $\mu$ some regularization scale. Similarly, the corresponding LEC that appears must also depend on $\mu$, thus making the observable scale-independent. This, however, also means that it is in general not possible to assign a definite value to the pion cloud contribution of an observable since shifts in the regularization scale allow one to shuffle strength from the long-range pion contribution to the shorter ranged contact term part. I refer the reader to Ref. [94], where the isovector charge radius of the proton 
is analyzed at one-loop order. It is shown that a modest change in the regularization scale from $\mu=0.8 \mathrm{GeV}$ to $\mu=1 \mathrm{GeV}$ (using conventional dimensional regularization) even leads to a sign change in the counterterm contribution. This explicitly shows that there is, of course, a contribution of the chiral (pion) physics to a given observable, but only the observable quantity (here: the isovector charge radius) is independent of the regularization scale. A more detailed discussion of this and related issues can be found in Refs. [95, 96].

\section{Summary and outlook}

The lessons learned and the take-home messages from this talk are:

- Resonances are defined as poles in the complex energy plane. To calculate their complex properties, one must locate the corresponding pole and then derive the resonance characteristics from suitably defined Laurent expansions around the pole position. Any other approach will in most cases lead to imprecise or even wrong results. Be aware of how many entries in the PDG tables have been obtained!

- The QCD spectrum is more than a collection of quark model states. The quark model should not be considered as a faithful representation of the QCD spectrum but rather as a simple approximation to a part of it. Note that the approximations underlying the conventional quark model are clearly better justifed for the heavy than for the light quarks.

- Structure formation in QCD ties nuclear and hadron physics together. This is most clearly seen through the appearance of molecular-type structures both in the nuclear as well as in the hadronic landscape. This was realized early by some researchers, see e.g. [97, 98].

- Lattice QCD is making progress in addressing complex resonance properties, even finding poles deep inside the complex plane, like for the lowest resonance in QCD, the $f_{0}(500)$, see e.g. $[99,100]$. However, the extrapolation into the complex plane must respect chiral symmetry. Otherwise, one can easily miss a possible two-pole structure which was first observed for the $\Lambda(1405)$ but appears to play an even bigger role for states involving charm and bottom quarks.

- EFTs are of utmost importance in pushing this program forward. This has become apparent in the discussion of finding poles in the complex plane. The explicit calculations of finite-volume effects in hadron decays are another important playground for EFTs and this program is pursued vigorously, see e.g. the talk by Pang [101].

\section{Acknowledgments}

I thank all my collaborators for sharing their insights on the various topics discussed here. Special thanks to Maxim Mai for supplying Fig. 3, and to Feng-Kun Guo, Bernard Metsch and Akaki Rusetsky for a careful reading of the manuscript. This work is supported in part by the DFG (Grant No. TRR110) and the NSFC (Grant No. 11621131001) through the funds provided to the Sino-German CRC 110 "Symmetries and the Emergence of Structure in QCD", by the Chinese Academy of Sciences (CAS) President's International Fellowship Initiative (PIFI) (grant no. 2018DM0034) and by VolkswagenStiftung (grant no. 93562). Computational resources for this project were provided by the Jülich Supercomputing Centre (JSC) at the Forschungszentrum Jülich and by RWTH Aachen.

\section{References}

[1] R. G. Moorhouse, quote from the 1960ties.

[2] F. K. Guo et al., Rev. Mod. Phys. 90, 015004 (2018).

[3] P. C. Bruns et al., Phys. Lett. B 697, 254 (2011).

[4] J. A. Oller et al., Phys. Lett. B 500, 263 (2001).

[5] A. Cieplý et al., Nucl. Phys. A 954, 17 (2016).

[6] M. Lüscher, Commun. Math. Phys. 105, 153 (1986).

[7] U. J. Wiese, Nucl. Phys. Proc. Suppl. 9, 609 (1989).

[8] K. Rummukainen et al., Nucl. Phys. B 450, 397 (1995).

[9] Bonn-Jülich-QCDSF collaboration, unpublished (2010).

[10] C. Morningstar, these proceedings.

[11] M. Petschlies, these proceedings.

[12] C. B. Lang et al., Phys. Rev. D 87, 054502 (2013).

[13] V. Verduci et al., PoS LATTICE 2014, 121 (2014).

[14] L. Leskovec et al., Few Body Syst. 59, 95 (2018).

[15] M. Werner et al., arXiv:1907.01237 [hep-lat].

[16] S. He et al., JHEP 0507, 011 (2005).

[17] M. Lage et al., Phys. Lett. B 681, 439 (2009).

[18] V. Bernard et al., JHEP 1101, 019 (2011).

[19] R. A. Briceño et al., Phys. Rev. D 88, 094507 (2013).

[20] R. A. Briceño et al., Phys. Rev. D 88, 034502 (2013).

[21] M. Döring et al., Eur. Phys. J. A 47, 139 (2011).

[22] M. Döring et al., Eur. Phys. J. A 48, 114 (2012).

[23] R. A. Briceño et al., Phys. Rev. D 95, 074510 (2017).

[24] J. M. M. Hall et al., Phys. Rev. Lett. 114, 132002 (2015).

[25] R. Molina et al., Phys. Rev. D 94, 056010 (2016) Addendum: [Phys. Rev. D 94, 079901 (2016)].

[26] G. Moir et al., JHEP 1610, 011 (2016).

[27] see http://pdg.lbl.gov/.

[28] N. Kaiser et al., Nucl. Phys. A 594, 325 (1995).

[29] E. Oset et al., Nucl. Phys. A 635, 99 (1998).

[30] M. F. M. Lutz et al., Nucl. Phys. A 700, 193 (2002).

[31] F. K. Guo et al., Phys. Lett. B 666, 251 (2008).

[32] L. Liu et al., Phys. Rev. D 87, 014508 (2013).

[33] M. Albaladejo et al., Phys. Lett. B 767, 465 (2017).

[34] D. Jido et al., Nucl. Phys. A 725, 181 (2003).

[35] E. E. Kolomeitsev et al., Phys. Lett. B 582, 39 (2004).

[36] F. K. Guo et al., Phys. Lett. B 641, 278 (2006).

[37] F. K. Guo et al., Eur. Phys. J. A 40, 171 (2009).

[38] Z. H. Guo et al., Phys. Rev. D 92, 094008 (2015).

[39] M. L. Du et al., Phys. Rev. D 98, 094018 (2018). 
[40] R. Aaij et al. [LHCb Collaboration], Phys. Rev. D 92, 012012 (2015).

[41] R. Aaij et al. [LHCb Collaboration], Phys. Rev. D 91, 092002 (2015) Erratum: [Phys. Rev. D 93, 119901 (2016)].

[42] R. Aaij et al. [LHCb Collaboration], Phys. Rev. D 94, 072001 (2016).

[43] M. L. Du et al., Phys. Rev. D 99, 114002 (2019).

[44] S. Weinberg, Phys. Rev. 137, B672 (1965).

[45] D. Morgan et al., Phys. Lett. B 258, 444 (1991).

[46] N. A. Tornqvist, Phys. Rev. D 51, 5312 (1995).

[47] V. Baru et al., Phys. Lett. B 586, 53 (2004).

[48] E. Braaten et al., Phys. Rev. D 76, 094028 (2007).

[49] F. Aceti et al., Phys. Rev. D 86, 014012 (2012).

[50] Z. H. Guo et al., Phys. Rev. D 93, 096001 (2016).

[51] M. Mai, these proceedings.

[52] E. Oset, these proceedings.

[53] R. Aaij et al. [LHCb Collaboration], Phys. Rev. Lett. 115072001 (2015).

[54] R. Aaij et al. [LHCb Collaboration], Phys. Rev. Lett. 122, 222001 (2019).

[55] J. J. Wu et al., Phys. Rev. Lett. 105, 232001 (2010).

[56] R. Chen et al., Phys. Rev. Lett. 115132002 (2015).

[57] T. J. Burns, Eur. Phys. J. A 51, 152 (2015).

[58] L. Roca et al., Phys. Rev. D 92, 094003 (2015).

[59] R. Chen et al., Phys. Rev. D 100, 011502 (2019).

[60] F. K. Guo et al., Phys. Rev. D 99091501 (2019).

[61] T. J. Burns et al., arXiv:1908.03528 [hep-ph] (2019).

[62] C. Bignamini et al., Phys. Rev. Lett. 103, 162001 (2009).

[63] M. Albaladejo et al., Chin. Phys. C 41, 121001 (2017).

[64] P. Artoisenet et al., Phys. Rev. D 81, 114018 (2010).

[65] F. K. Guo et al., Commun. Theor. Phys. 61, 354 (2014).

[66] F. K. Guo et al., Eur. Phys. J. C 74, 3063 (2014).

[67] F. K. Guo et al., Phys. Rev. D 88, 054007 (2013).

[68] F. K. Guo et al., JHEP 1405, 138 (2014).

[69] G. Bauer [CDF Collaboration], Int. J. Mod. Phys. A 20, 3765 (2005).
[70] S. Chatrchyan et al. [CMS Collaboration], JHEP 1304, 154 (2013).

[71] S. Fleming et al., Phys. Rev. D 76, 034006 (2007).

[72] P. F. Bedaque et al., Ann. Rev. Nucl. Part. Sci. 52, 339 (2002).

[73] D. L. Canham et al., Phys. Rev. D 80014009 (2009).

[74] R. G. Stuart, in "Z0 Physics”, ed. J. Tran Thanh Van (Editions Frontieres, Gif-sur-Yvette, 1990), p. 41.

[75] A. Denner et al., Nucl. Phys. B 560, 33 (1999).

[76] D. Djukanovic et al., Phys. Lett. B 680, 235 (2009).

[77] A. Denner et al., Nucl. Phys. Proc. Suppl. 160, 22 (2006).

[78] W. Beenakker et al., Nucl. Phys. B 338, 349 (1990).

[79] J. Gegelia et al., Phys. Lett. B 763, 1 (2016).

[80] H. B. Tang et al., Phys. Lett. B 387, 9 (1996).

[81] H. Krebs et al., Phys. Lett. B 683, 222 (2010).

[82] M. J. G. Veltman, Physica 29, 186 (1963).

[83] D. Siemens et al., Phys. Lett. B 770, 27 (2017).

[84] D. L. Yao et al., JHEP 1605, 038 (2016).

[85] J. Gegelia et al., Phys. Lett. B 760, 736 (2016.

[86] B. Borasoy et al., Phys. Lett. B 641, 294 (2006).

[87] D. Djukanovic et al., Phys. Lett. B 690, 123 (2010).

[88] B. Long et al., Nucl. Phys. A 870-871, 72 (2011).

[89] S. R. Beane et al., J. Phys. G 31, 921 (2005).

[90] M. Gelenava, Eur. Phys. J. A 54, 88 (2018).

[91] V. Burkert, these proceedings.

[92] H. Pagels, Phys. Rept. 16, 219 (1975).

[93] V. Bernard et al., Phys. Rev. Lett. 67, 1515 (1991).

[94] V. Bernard et al., Nucl. Phys. A 635, 121 (1998) [Erratum-ibid. A 642, 563 (1998) 563].

[95] U.-G. Meißner, AIP Conf. Proc. 904, 142 (2007).

[96] E. M. Henley et al., 100 Years of Subatomic Physics (World Scientific, Singapore, 2013) 199-229.

[97] M. B. Voloshin et al., JETP Lett. 23, 333 (1976)

[Pisma Zh. Eksp. Teor. Fiz. 23, 369 (1976)].

[98] N. A. Tornqvist, Z. Phys. C 61, 525 (1994).

[99] R. A. Briceño, et al., Phys. Rev. Lett. 118, 022002 (2017).

[100] D. Guo et al., Phys. Rev. D 98, 014507 (2018).

[101] J.-Y. Pang, these proceedings. 